\title{
An Infinite Elastic Plate Weakened by a Generalized Curvilinear Hole and Goursat Functions
}

\author{
Mohamed Abdellah Abdou1, Azhar Rashad Jan² \\ ${ }^{1}$ Department of Mathematics, Alexandria University, Alexandria, Egypt \\ ${ }^{2}$ Department of Mathematics, Umm Al-Qura University, Mecca, KSA \\ Email: abdella777@yahoo.com, azhaarjaan@ymail.com
}

Received 23 December 2013; revised 23 January 2014; accepted 2 February 2014

Copyright @ 2014 by authors and Scientific Research Publishing Inc.

This work is licensed under the Creative Commons Attribution International License (CC BY). http://creativecommons.org/licenses/by/4.0/

(c) (i) Open Access

\begin{abstract}
Complex variables method has been used to solve the first and second fundamental problems for an infinite plate weakened by a generalized curvilinear hole $C$. The curvilinear hole is conformally mapped on the domain outside or inside a unit circle $\gamma$ using a general rational mapping function with complex constants. Many special and new cases are derived from this work. Some of the work of the previous authors in this domain will be considered as special cases of this paper. Also the interesting cases when the shape of the hole takes different famous shapes are included. The components of stresses for some examples are obtained.
\end{abstract}

\section{Keywords}

Goursat Functions; Conformal Mapping; Curvilinear Hole; Stress Components

\section{Introduction}

The boundary value problems for isotropic homogeneous performed infinite plates have been discussed by several authors: see Colton and Kress [1], Popov [2], Noda et al. [3] and Schinzinger and Laura [4]. Some authors used Laurent's theorem to express the solution in the series form, see England [5], Parkus [6] and Kalandiya [7]. Others used complex variables method of Cauchy integrals to express the solution of the boundary value problems in the form of two complex potential functions, Goursat functions, by using many rational mappings, see Muskhelishvili [8], El-Sirafy and Abdou [9], Abdou and Khar-Eldin [10], Abdou and Khamis [11], Abdou [12] and Abdou et al. [13]. In all previous works, the coefficients of the rational mappings were real.

It is worth mentioning that Exadaktylos and Stavropoulou [14] and Exadaktylos et al. [15] considered rational 
mapping functions with complex constants that conformally maps the holes inside a unit circle, using Laurent's method. Also Abdou and Asseri [16] [17] considered more general rational mapping functions with complex constants that conformally maps the holes outside and inside a unit circle, using Cauchy singular method. All the previous four works will be considered as special cases of this work.

It is known that, see Muskhelishvili [8], the first and second fundamental problems in the plane theory of elasticity are equivalent to finding two analytic functions $\phi_{1}(z)$ and $\psi_{1}(z)$ of one complex argument $z=x+\mathrm{i} y$. These analytic potential functions, Goursat functions, must satisfy the boundary conditions

$$
k \phi_{1}(t)-t \overline{\phi_{1}^{\prime}(t)}-\overline{\psi_{1}(t)}=f(t),
$$

where, $k=-1$ and $f(t)$ is a given function of stress, for the first fundamental problem. While

$k=\chi=\frac{\lambda+3 \mu}{\lambda+\mu}>1$, and $f(t)=2 \mu g(t)$ is a given function of the displacement for the second fundamental problem; $\lambda, \mu$ are called the Lame's constants and $t$ denotes the affix of a point on the boundary.

In terms of the rational mapping function $z=c w(z), c>0, w^{\prime}(z)$ does not vanish or become infinite for $|\zeta|>1$, the infinite region outside a closed contour conformally mapped outside the unit circle $\gamma$. The two complex potential functions $\phi_{1}(z), \psi_{1}(z)$, in this case, take the forms

$$
\begin{aligned}
& \phi_{1}(z)=-\frac{X+\mathrm{i} Y}{2 \pi(1+\chi)} \ln \zeta+c \Gamma \zeta+\phi(\zeta) \\
& \psi_{1}(z)=\frac{\chi(X-\mathrm{i} Y)}{2 \pi(1+\chi)} \ln \zeta+c \Gamma^{*} \zeta+\psi(\zeta),
\end{aligned}
$$

where $X, Y$ are the components of the resultant vector of all external forces acting on the boundary and $\Gamma, \Gamma^{*}$ are complex constants. The two complex functions $\phi(\zeta)$ and $\psi(\zeta)$ are single valued analytic functions within the region outside the unit circle and $\phi(\infty)=\psi(\infty)=0$. For the first fundamental problem, we have $X=Y=0$, and $\Gamma=\bar{\Gamma}$.

In the absence of body forces, Muskhelishvili [8] has considered the stress components in the plane theory of elasticity in the form

$$
\begin{gathered}
\sigma_{x x}+\sigma_{y y}=4 \operatorname{Re}\left\{\phi^{\prime}(z)\right\}, \\
\sigma_{y y}-\sigma_{x x}+2 \mathrm{i} \sigma_{x y}=2\left[\bar{z} \phi^{\prime \prime}(z)+\psi^{\prime}(z)\right]
\end{gathered}
$$

In this work, the complex variables method will be applied to solve the first and second fundamental problems for an infinite plate with a generalized curvilinear hole $C$ conformally mapped on the domain outside a unit circle $\gamma$ by the generalized rational mapping function

$$
z=\frac{d \zeta+m \zeta^{-\ell}}{1-n \zeta^{-\ell}},(|n|<1, z=x+\mathrm{i} y, i=\sqrt{-1})
$$

where $d=d_{1}+\mathrm{i} d_{2}, m=m_{1}+\mathrm{i} m_{2}, n=n_{1}+\mathrm{in}_{2}, \ell=1,2, \cdots, P$; and $|m / d|$ is a parameter restricted such that $z^{\prime}(\zeta)$ does not vanish or become infinite outside the unit circle $\gamma(|\zeta|>1)$. The holes take different famous shapes which make these studies applicable for many phenomena throughout the nature like tunnels, caves, excavations in soil or rock, etc. Moreover, the results of Goursat functions when the transformation mapping (6) is conformally mapped inside the unit circle $\gamma(|\zeta|<1)$ are discussed and obtained.

Also, many applications for the first and second fundamental problems are considered and the components of stress and strain have been obtained and plotted to investigate their physical meaning. Moreover, computer work using maple 9.5 has been used in applications to give the shapes of holes and curves of stresses with some calculations of stresses at their important points.

\section{The Rational Mapping}

The physical interest of the mapping (6) comes from its special cases and its different shapes of holes that can be obtained, see Figures 1-6. 
From the rational mapping we can discuss the following:

1) The number of the holes corners is subjected to $\ell$ 's values. There are given by $\ell+1$.

2) The shape of the hole depending on the values of $n$ 's and $m$ 's.

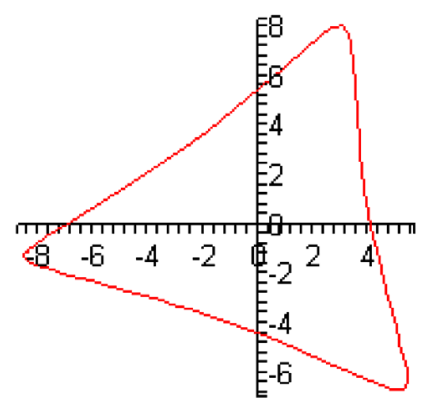

Figure 1. $\ell=2, n=(0,0), m=(1,2), d=(2,6)$.

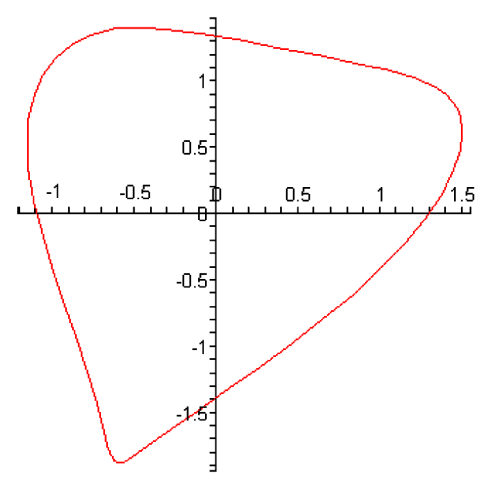

Figure 2. $\ell=2, n=(0.1,0.1), m=(0.2,-0.2), d=(1,1)$.

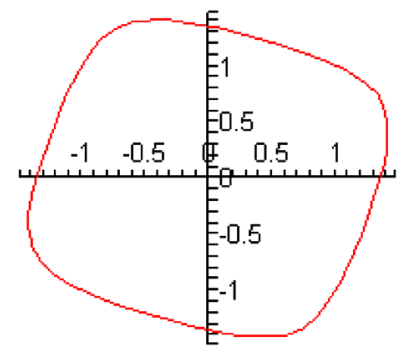

Figure 3. $\ell=3, n=(0.001,0.001), m=(0.1,-0.05), d=(1,1)$.

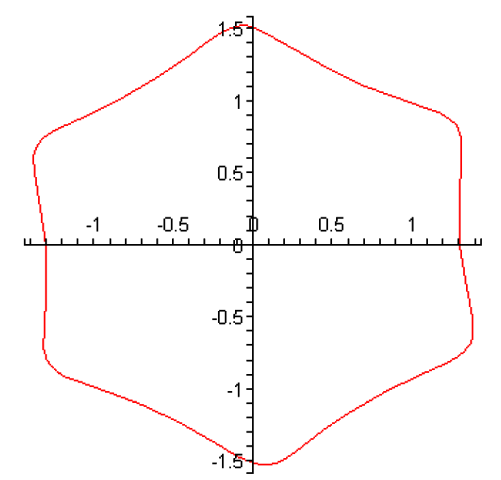

Figure 4. $\ell=5, n=(0.001,0.001), m=(0.1,-0.05), d=(1,1)$. 


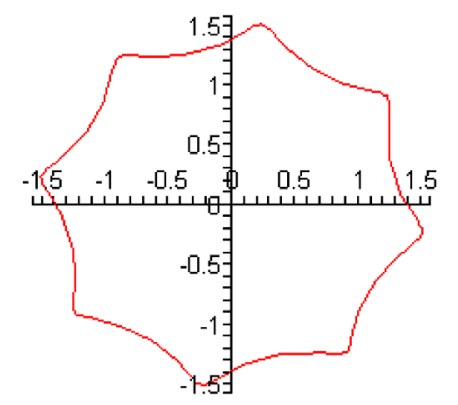

Figure 5. $\ell=7, n=(0.001,0.001), m=(0.1,-0.05), d=(1,1)$.

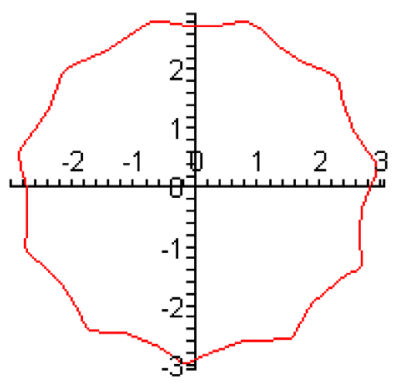

Figure 6. $\ell=10, n=(0.009,0.007), m=(0.1,-0.05), d=(2,2)$.

3) Entering none zero values of the complex constants $m$ and $d$ never gives symmetric graphs. While, entering zero values for all imaginary parts of both $m$ and $d$, we get symmetric shapes around the $x$-axis. On the other hand, entering zero values for all real parts of both $m$ and $d$, we get symmetric shape around the $y$-axis.

4) The complex constant $m$ works on circling the shape from the symmetry situation and the circling angle is given by $\theta=\tan ^{-1}\left(m_{2} / m_{1}\right), \quad m=m_{1}+i m_{2}$. Positive values of $\theta$ means that the circling will be in the positive direction i.e. in the anti clockwise direction and for negative values the circling will be in the negative direction i.e. in clockwise direction.

5) Using the rational mapping function $z_{1}=z^{\frac{\pi_{i}}{4}}, \mathrm{i}=\sqrt{-1}$, enables us to enter none zero vales of $d, m$ and $n$ complex constants and getting symmetric shapes around the $x$-axis. But by substituting zero values for real parts, we get the same shapes that have been gotten by using the rational mapping $z(\zeta)$, and the invested shapes of it will be given by substituting zero values for the imaginary parts of the complex constants $d, m$ and $n$.

6) The complex constant $d$ works on expanding the corners of the hole shape.

\section{Goursat Functions}

In this section, we use the transformation mapping (6) in the boundary conditions (1), and complex variables method, Cauchy method, to obtain a closed form expression for the Goursat functions $\phi(\zeta)$ and $\psi(\zeta)$ respectively. Therefore, we write

$$
\frac{\omega(\zeta)}{\omega^{\prime}\left(\zeta^{-1}\right)}=\alpha(\zeta)+\overline{\beta(\zeta)},
$$

where

$$
\begin{gathered}
\alpha(\zeta)=\frac{h}{\zeta^{\ell}-n}, \\
h=\frac{\left(d n^{v}+m\right)(1-n \bar{n})^{2}}{\bar{d}\left[1-n \bar{n}(1+\ell)-l \bar{m} n^{v}\right]},\left(v=1+\frac{1}{\ell}\right),
\end{gathered}
$$


and $\beta(\zeta)$ is a regular function for $|\zeta|>1$.

Using (7) in the boundary conditions (1) and on $\zeta=\sigma$, we get

$$
k \phi(\sigma)-a(\sigma) \overline{\phi^{\prime}(\sigma)}-\overline{\psi_{*}(\sigma)}=f_{*}(\sigma)
$$

where

$$
\begin{gathered}
\psi_{*}(\zeta)=\psi(\zeta)+\beta(\zeta) \phi^{\prime}(\zeta) \\
f_{*}(\zeta)=F(\zeta)-d k \Gamma \zeta+\frac{\bar{d} \bar{\Gamma}^{*}}{\zeta}+N(\zeta)[\alpha(\zeta)+\overline{\beta(\zeta)}] \\
N(\zeta)=\bar{d} \bar{\Gamma}-\frac{X-\mathrm{i} Y}{2 \pi(1+\chi)} \zeta
\end{gathered}
$$

and

$$
F(\zeta)=f(t)
$$

The function $F(\zeta)$ with its derivatives must satisfy the Hölder condition. Multiplying both sides of (10) by $\frac{1}{2 \pi \mathrm{i}} \frac{1}{\sigma-\zeta}$ and integrating with respect to $\sigma$ on $\gamma$, we have

$$
-k \phi(\zeta)=A(\zeta)-\frac{\bar{d} \overline{\Gamma^{*}}}{\zeta}-\frac{h}{\zeta^{\ell}-n}\left(b+N\left(n^{\nu-1}\right)\right)
$$

where

$$
A(\zeta)=-\frac{1}{2 \pi \mathrm{i}} \sum_{j=0}^{\infty} \zeta^{-(j+1)} \int_{\gamma} \sigma^{j} F(\sigma) \mathrm{d} \sigma,|\zeta|>1
$$

and the complex constant $b$, will be determined, is given by

$$
\frac{1}{2 \pi \mathrm{i}} \int_{\gamma} \frac{\alpha(\sigma) \overline{\phi^{\prime}(\sigma)}}{\sigma-\zeta} \mathrm{d} \sigma=\frac{h b}{n-\zeta^{\ell}} .
$$

Differentiating (15) with respect to $\zeta$, then using the result in (17), the complex constant $b$ takes the form

$$
b=\frac{k E-\eta \bar{h} \bar{E}}{k^{2}-\eta \bar{\eta} h \bar{h}}
$$

where

$$
E=-\left[\overline{A^{\prime}\left(n^{\nu-1}\right)}+d \Gamma^{*} n^{2(v-1)}+\eta \bar{h} \overline{N\left(n^{v-1}\right)}\right], \quad \eta=\frac{n^{2(v-1)}}{\left(\bar{n}^{(v-1)} n^{(v-1)}-1\right)^{2}}
$$

Also, the function $\psi(\zeta)$ can be determined from (1) in the form

$$
\psi(\zeta)=\frac{k \bar{d} \bar{\Gamma}}{\zeta}-\frac{\omega\left(\zeta^{-1}\right)}{\omega^{\prime}(\zeta)} \varphi_{*}(\zeta)+\frac{\bar{h} \zeta^{\ell}}{1-\bar{n} \zeta^{\ell}} \varphi_{*}\left(n^{1-v}\right)+B(\zeta)-B
$$

where

$$
\phi_{*}(\zeta)=\phi^{\prime}(\zeta)+\overline{N(\zeta)}, B(\zeta)=\frac{1}{2 \pi \mathrm{i}} \int_{\gamma} \frac{\overline{F(\sigma)}}{\sigma-\zeta} \mathrm{d} \sigma, B=\frac{1}{2 \pi \mathrm{i}} \int_{\gamma} \frac{\overline{F(\sigma)}}{\sigma} \mathrm{d} \sigma
$$

The two formulas (15) and (19) are representing the Goursat functions for the first and second fundamental problems for an infinite elastic plate weakened by The two formulas (15) and (19) are representing the Goursat functions for the first and second fundamental problems for an infinite elastic plate weakened by a generalized 
curvilinear hole $C$, that can be transformed outside a unit circle $\gamma$ by the rational mapping (6).

An important new case for discussion is using the transformation mapping

$$
z=\frac{d \zeta^{-1}+m \zeta^{\ell}}{1-n \zeta^{\ell}},|n|<1
$$

This mapping function, when $z^{\prime}(\zeta) \neq 0 ;|\zeta|<1$, transforms the points in the $z$-plane inside the unit circle $\gamma$ in $\zeta$-plane. And, in this case, the Goursat functions, become

$$
\begin{gathered}
k \phi(\zeta)=-A\left(\zeta^{-1}\right)+\bar{d} \overline{\Gamma^{*}} \zeta+\frac{h \zeta^{\ell}}{1-n \zeta^{\ell}}\left(b+N\left(n^{\nu-1}\right)\right), \\
\psi(\zeta)=k \bar{d} \bar{\Gamma} \zeta-\frac{\omega(\zeta)}{\omega^{\prime}\left(\zeta^{-1}\right)} \varphi_{*}\left(\zeta^{-1}\right)+\frac{\bar{h}}{\zeta^{\ell}-n} \varphi_{*}\left(n^{1-v}\right)+B\left(\zeta^{-1}\right)-B
\end{gathered}
$$

\section{Special Cases}

Here, we discuss the following:

1) By considering the reality of the constants of the mapping (1.6), the Goursat functions, in this case, are agree with work of Abdou and Khar-Eldin [10] of Equations (15) and (19), on notation the difference in notation.

2) When $\ell=1$ and $m=\{0,0\}$ and for finite expansion, the transformation mapping (6), in this case, becomes

$$
Z=d \zeta+\sum_{J=1}^{M} n_{J} \zeta^{-J}, d, n_{J} \text { are complex constants }
$$

The Goursat functions, in this case, become

$$
\begin{gathered}
k \phi(\zeta)=-A(\zeta)+\frac{\bar{d} \overline{\Gamma^{*}}}{\zeta} \\
+\frac{1}{|d|^{2}} \sum_{J=1}^{M} \sum_{k=0}^{J-1} \frac{1}{(J-1-k) !} \frac{n_{J} \overline{\varphi^{J-k}(0)}}{\zeta^{1+k}}+\frac{1}{|\bar{d}|^{2}} \sum_{J=1}^{M} \frac{n_{J}}{\zeta^{J}} N(0) \\
\psi(\zeta)=\frac{k \bar{d} \bar{\Gamma}}{\zeta}-\frac{\omega\left(\zeta^{-1}\right)}{\omega^{\prime}(\zeta)} \phi_{*}(\zeta)+\frac{\bar{d}}{|d|^{2}} \sum_{J=1}^{M} \overline{n_{J}} \zeta^{J} \overline{N(0)}+B(\zeta)-B \\
+\frac{\bar{d}}{|d|^{2}} \sum_{J=1}^{M} \sum_{k=0}^{J-1} \frac{1}{(J-1-k) !} \overline{n_{J}} \varphi^{J-k}(0) \zeta^{(k+1)}
\end{gathered}
$$

The results of the two formulas (24) and (25) are in agreement with the work of Abdou and Asseri [16], on noting the difference in notation.

3) When $\ell=1$, the transformation mapping (6) becomes

$$
z=\frac{d \zeta+m \zeta^{-1}}{1-n \zeta^{-1}}, \quad(d, m, n \text { are complex constants). }
$$

The Goursat functions, in this case, of the two formulas (15) and (19) agree with the all results of Abdou and Asseri [17].

4) In the mapping function (20) if we let $m=0$, then for finite expansion, we will have the following mapping function

$$
z=d \zeta^{-1}+\sum_{J=1}^{M} n_{J} \zeta^{J-1}(|\zeta|<1)
$$

with the corresponding Goursat functions 


$$
\begin{gathered}
-k \phi(\zeta)=A\left(\zeta^{-1}\right)+\bar{d} \overline{\Gamma^{*}} \zeta-\frac{1}{|d|^{2}} \sum_{J=1}^{M} \sum_{k=0}^{J-1} \frac{1}{(J-1-k) !} n_{J} \overline{\varphi^{(J-k)}}(0) \zeta^{1+k}-\frac{1}{|d|^{2}} \sum_{J=1}^{M} N(0) n_{J} \zeta^{J} \\
\psi(\zeta)= \\
k \bar{d} \bar{\Gamma} \zeta-\frac{\omega(\zeta)}{\omega^{\prime}\left(\zeta^{-1}\right)} \phi_{*}\left(\zeta^{-1}\right)+\frac{\bar{d}}{|d|^{2}} \sum_{J=1}^{M} N(0) \overline{n_{J}} \zeta^{-J}+B\left(\zeta^{-1}\right)-B \\
+\frac{\bar{d}}{|d|^{2}} \sum_{J=1}^{M} \sum_{k=0}^{J-1} \frac{1}{(J-1-k) !} \overline{\phi^{J-k}}(0) \zeta^{-(k+1)}
\end{gathered}
$$

The three Formulas (27)-(29) are equivalent to those derived by Exadaktylos and Stavropoulou [14], where they used Laurent's theorem, after considering in (27)-(29) the following special cases: $M=3, k=-1, \Gamma=\bar{\Gamma}$, $X=Y=0$ and $f=2 \lambda(\Gamma+P) z-\lambda \Gamma^{*} \bar{z}, 0 \leq \lambda \leq 1$. The constant $\lambda$ is called the situ stress relief factor i.e. for $\lambda$ $=0$ no excavation has been occurred and for $\lambda=1$, the tunnels is fully excavated. More information and physical meaning for $\lambda$ can be found in the work of Exadaktylos and Stavropoulou [14].

5) Also, in (27), if we allow the index inside the summation sign to take the form $z=\frac{d}{\zeta}+\sum_{J=1}^{M} n_{2 J-1} \zeta^{2 J-1}$, in addition to the consideration of the reality all constants. Then using in (28) and (29) the following: $k=-1$, $\Gamma=\bar{\Gamma}, X=Y=0$ and $f(z)=-P z ; P$ is the intensity of the tensile stress and $z$ is the above rational mapping, the results will agree with the work of Exadaktylos et al. [15].

\section{Applications}

1) For $k=-1, G=P / 4, \Gamma^{*}=-\frac{1}{2} P \mathrm{e}^{-2 \mathrm{i} \vartheta}$ and $X=Y=f=0$, we have the Goursat functions in the form

$$
\begin{gathered}
\phi(\zeta)=\frac{1}{2} \bar{d} P \mathrm{e}^{2 \mathrm{i} \vartheta} \zeta^{-1}-\frac{h}{\left(\zeta^{\ell}-n\right)}\left(b+N\left(n^{\nu-1}\right)\right), \\
\psi(\zeta)=-\frac{1}{4} \bar{d} P \zeta^{-1}-\frac{\omega\left(\zeta^{-1}\right)}{\omega^{\prime}(\zeta)} \phi_{*}(\zeta)+\frac{\bar{h} \zeta^{\ell}}{1-\bar{n} \zeta^{\ell}} \phi_{*}\left(n^{1-v}\right) .
\end{gathered}
$$

The complex constant $b$ has been determined by Equation (18) and its value was calculated by using Maple 9.5. Here, we have the Goursat functions for an infinite plate weakened by a curvilinear hole $C$ which is free from stresses. The plate stretched at infinity by the application of a uniform tensile stress of intensity $P$, making an angle $\theta$ with the x-axis.

For $\ell=2, n=0.1+0.1 \mathrm{i}, m=0.2-0.2 \mathrm{i}, d=1+\mathrm{i}, \quad P=1 / 4$ and $\vartheta=\pi / 4, \quad \pi \approx 3.1415$ the relation between the stress components $\sigma_{x x}, \sigma_{y y}, \sigma_{x y}$ and the angle $\theta$ are considered in Figures 7-9.

2) For $k=-1, X=Y=\Gamma=\Gamma^{*}=0$ and $f=P t, P$ is a real constant, we have

$$
\begin{gathered}
\phi(\zeta)=-P \frac{d n^{v}+m}{\left(\zeta^{\ell}-n\right)}-\frac{h b}{\left(\zeta^{\ell}-n\right)}, \\
\psi(\zeta)=-\frac{\omega\left(\zeta^{-1}\right)}{\omega^{\prime}(\zeta)} \varphi^{\prime}(\zeta)+\frac{\bar{h} \zeta^{\ell}}{1-\bar{n} \zeta^{\ell}} \varphi^{\prime}\left(n^{1-v}\right)-\frac{\bar{d} P}{\zeta} .
\end{gathered}
$$

Thus, (32) and (33) give the solution of the first fundamental problem for an isotropic infinite plate with a curvilinear hole, when there are no external forces and the edge of the hole is subject to a uniform pressure $P$.

If in application (2) we write $f(t)=-\mathrm{i} T t$, we have the case of the first fundamental problem, when the edge of the hole is subject to uniform tangential stress $T$. The Goursat functions, in this case, are obtained directly from the two formulas (32) and (33) by putting $-\mathrm{i} T$ instead of $P$.

For $\ell=2, n=0.1+0.1 \mathrm{i}, m=0.2-0.2 \mathrm{i}, d=1+\mathrm{i}$ and $P=1 / 4$ the relation between the stress components $\sigma_{x x}, \sigma_{y y}, \sigma_{x y}$ and the angle $\theta$, using Maple 9.5 are considered in Figures 10-12. 


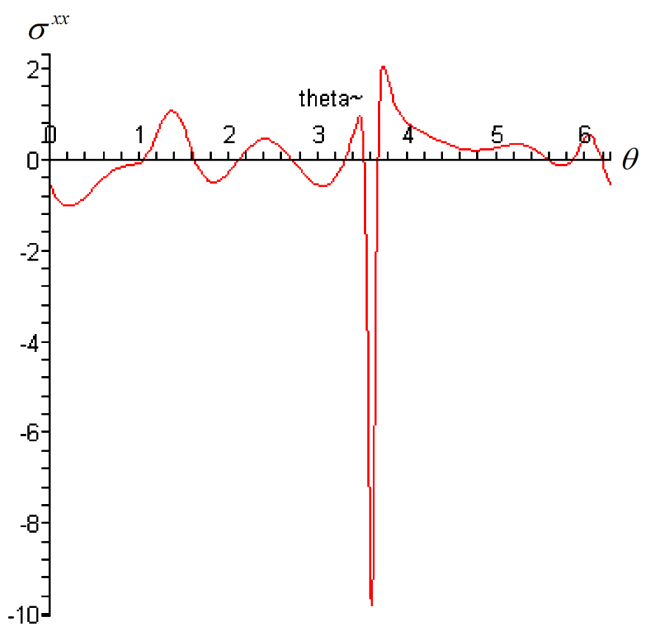

Figure 7. Max. and Min. values of $\sigma_{x x}$ are [2.09232, [ $\left.\left.\theta=3.7365\right]\right]$, $[-0.49508,[\theta=1.82742]]$.

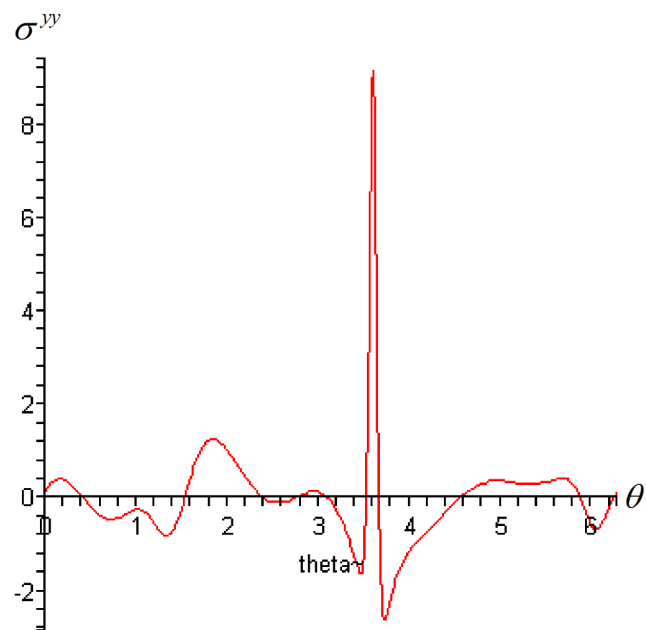

Figure 8. Max. and Min. values of $\sigma_{v v}$ are [1.24503, [ $\left.\left.\theta=1.84222\right]\right]$, $[-2.63598,[\theta=0.73521]]$.

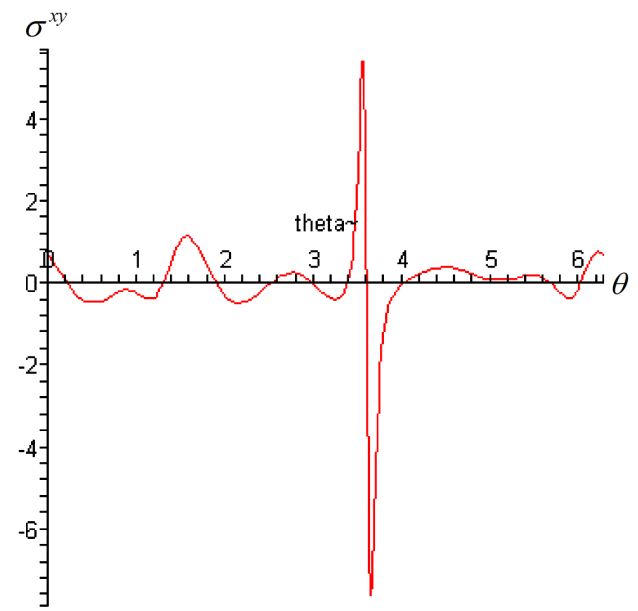

Figure 9. Maximum value of $\sigma_{x y}$ is [0.25045, [ $\left.\left.\theta=2.75306\right]\right]$, Minimum value of $\sigma_{x y}$ is $[-7.61349,[\theta=3.65448]]$. 


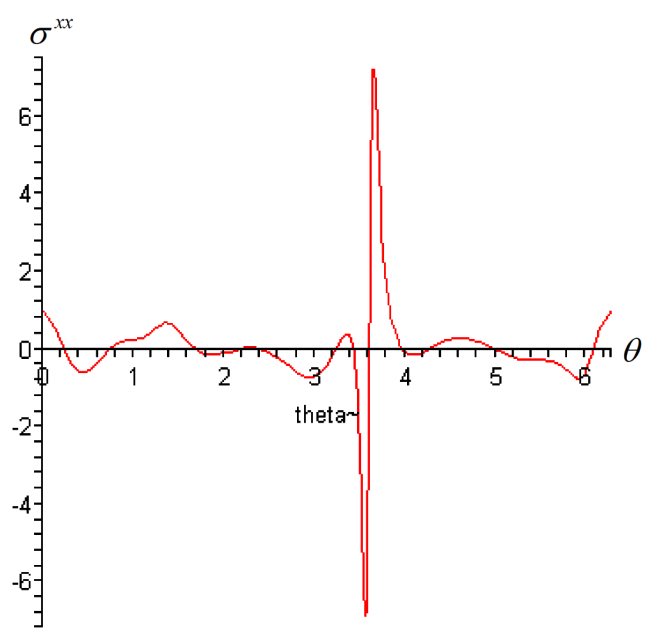

Figure 10. Max. and Min. values of $\sigma_{x x}$ are [7.26497, [ $\left.\left.\theta=3.66684\right]\right]$, $[-0.74520,[\theta=2.95389]]$.

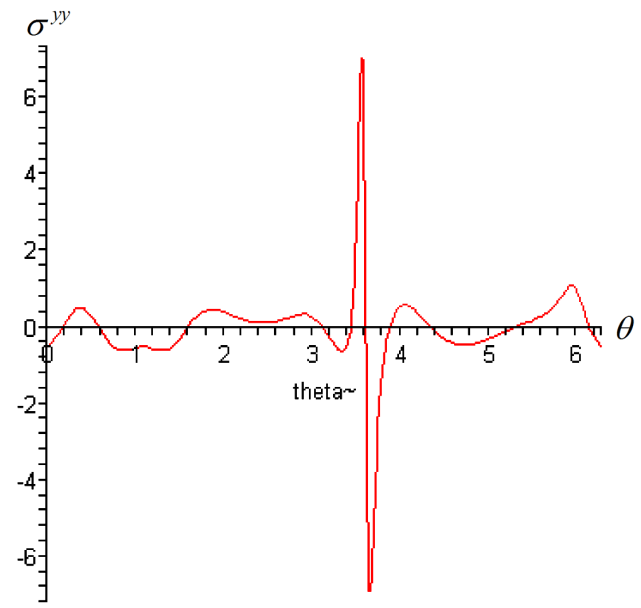

Figure 11. Max. and Min. values of $\sigma_{y y}$ are [0.45410, [ $\left.\left.\theta=1.87226\right]\right]$, $[-6.94161,[\theta=3.66649]]$.

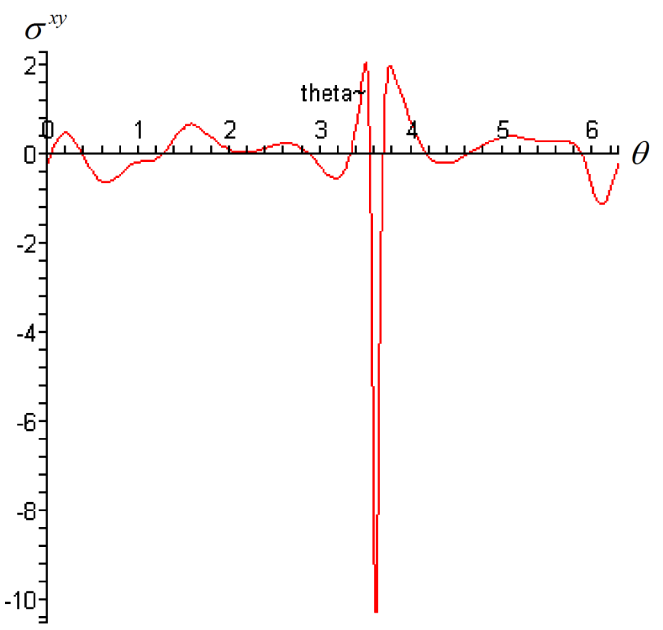

Figure 12. Maximum value of $\sigma_{x y}$ is [1.99460, $\left.[\theta=3.77003]\right]$, Minimum value of $\sigma_{x y}$ is $[-0.63053,[\theta=0.63788]]$. 
3) For $k=\chi, \Gamma=\frac{1}{4} P, \Gamma^{*}=-\frac{1}{2} P \mathrm{e}^{-2 \mathrm{i} \vartheta}, X=Y=0, f=2 \mu g(t)$ and $g(t)=\mathrm{i} t \varepsilon$, we have

$$
\begin{gathered}
\chi \phi(\zeta)=2 \mathrm{i} \mu \varepsilon \frac{d n^{v}+m}{\zeta^{\ell}-n}+\frac{\bar{d} \Gamma^{*}}{\zeta}+\frac{h}{\zeta^{\ell}-n}\left(b+\frac{\bar{d} P}{4}\right), \\
\psi(\zeta)=\bar{d}\left(\frac{P}{4} \chi+2 \mathrm{i} \mu \varepsilon\right) \zeta^{-1}-\frac{\omega\left(\zeta^{-1}\right)}{\omega^{\prime}(\zeta)} \phi_{*}(\zeta)+\frac{\bar{h} \zeta^{l}}{1-\bar{n} \zeta^{l}} \phi_{*}\left(n^{1-v}\right),
\end{gathered}
$$

where

$$
\phi_{*}(\zeta)=\phi^{\prime}(\zeta)+\frac{1}{4} d P
$$

Here, we have the case of uni-directional tension of an infinite plate with a rigid curvilinear centre. The constant $\varepsilon$, which represents the angle of rotation, can be determined from the condition that the resultant moment of the forces, acting on the curvilinear centre from the surrounding material, must vanish i.e.

$$
M=\operatorname{Re}\left\{\int\left[\psi(\zeta)-\frac{d}{2} P \mathrm{e}^{-2 \mathrm{i} \vartheta} \zeta\right] \omega^{\prime}(\zeta) \mathrm{d} \zeta\right\}=0 .
$$

Hence, we have

$$
\begin{gathered}
\varepsilon=\operatorname{Re} \frac{p(m+n \bar{n})[1-(m+2) n \bar{n}][1+\chi] \sin (2 \vartheta)}{4 \mu[m(m+n \bar{n})+\chi\{1-n \bar{n}(m+2)\}]}(\text { for } \ell=1), \\
\varepsilon=\operatorname{Re} \frac{P(1-n \bar{n})^{2} \sin (2 \vartheta)}{4 \mu \chi\left(1-2 m n^{3 / 2}-3 n \bar{n}\right)}\left\{n+\frac{\left[n\left(m+n^{3 / 2}\right)+(1+n)(1+\bar{n})+\left(2 m n^{3 / 2}+3 n \bar{n}-1\right) \chi\right] J_{2}}{2 n \bar{n}\left(m+n^{3 / 2}\right)^{2}\left(-n^{2} \bar{n}^{-2}+2 n \bar{n}+7\right) J_{3}}\right\}(\text { for } \ell=2)
\end{gathered}
$$

where

$$
\begin{aligned}
& J_{2}= 2 n \bar{n}(n \bar{n}-3)\left(m+n^{3 / 2}\right)+n\left(2 m n^{3}+3 n \bar{n}-1\right)\left(m+n^{3 / 2}\right)^{-1} \\
&+\left[\frac{4}{\bar{n}}\left(2 m n^{3 / 2}+3 n \bar{n}-1\right) \chi\right] \\
& J_{3}=\left(1-2 m n^{3 / 2}-3 n \bar{n}\right)\left(\frac{2 m}{\bar{\eta}}+n+n^{2} \bar{n}\right)
\end{aligned}
$$

For $\theta \quad \ell=1, n=0.1+0.1 \mathrm{i}, m=0.2-0.2 \mathrm{i}, d=1+\mathrm{i}, P=1 / 4, \vartheta=\pi / 4, \chi=2$ and $\mu=0.6$ the relation between the stress components $\sigma_{x x}, \sigma_{y y}, \sigma_{x y}$ and the angle $\theta$ are considered in Figures 13-15.

From the previous results, we can establish the following

Case (1): In the case of Bi-axial tension, we have $k=\chi, X=Y=0, \Gamma=\bar{\Gamma}=\frac{1}{2} P, \Gamma^{*}=0, \ell=1, \varepsilon=0$.

Hence, we get

$$
\begin{gathered}
\phi(\zeta)=\frac{1}{\chi} \frac{h}{(\zeta-n)}\left(b+\frac{\bar{d} P}{2}\right), \\
\psi(\zeta)=\frac{\chi \bar{d} P}{2 \zeta}-\frac{\omega\left(\zeta^{-1}\right)}{\omega^{\prime}(\zeta)} \phi_{*}(\zeta)+\frac{\bar{h} \zeta}{1-\bar{n} \zeta} \phi_{*}\left(n^{-1}\right),
\end{gathered}
$$

where

$$
\phi_{*}(\zeta)=\phi(\zeta)+\frac{1}{2} d P
$$


M. A. Abdou, A. R. Jan

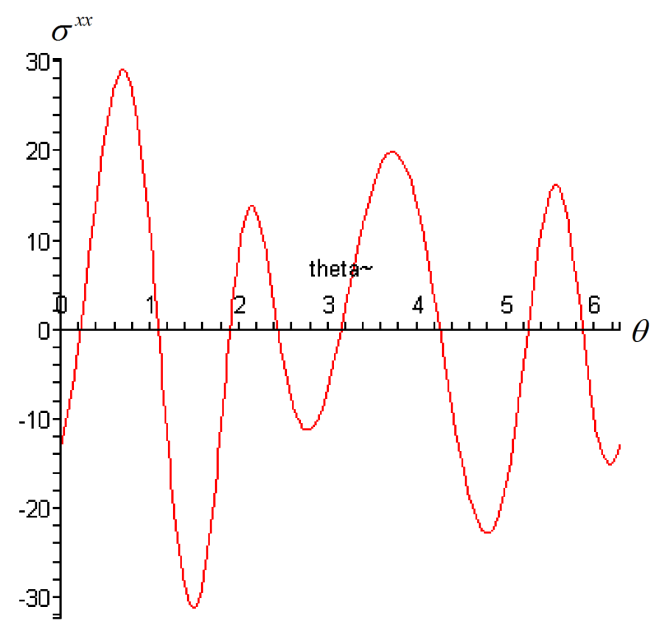

Figure 13. Max. and Min. values of $\sigma_{x x}$ are [19.94724, $[\theta=$ 3.72527]], [-31.09023, [ $\theta=1.48899]]$.

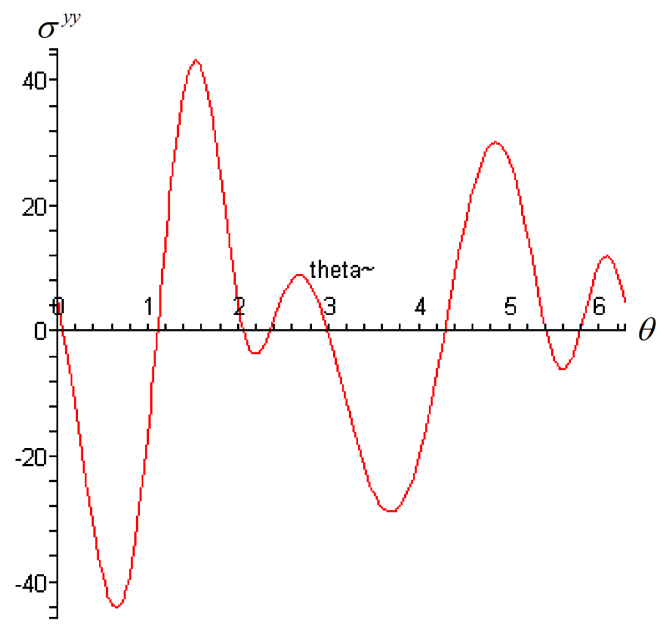

Figure 14. Max. and Min. values of $\sigma_{y y}$ are [43.21619, [ $\theta=$ $1.52536]],[-28.86760,[\theta=3.68310]]$.

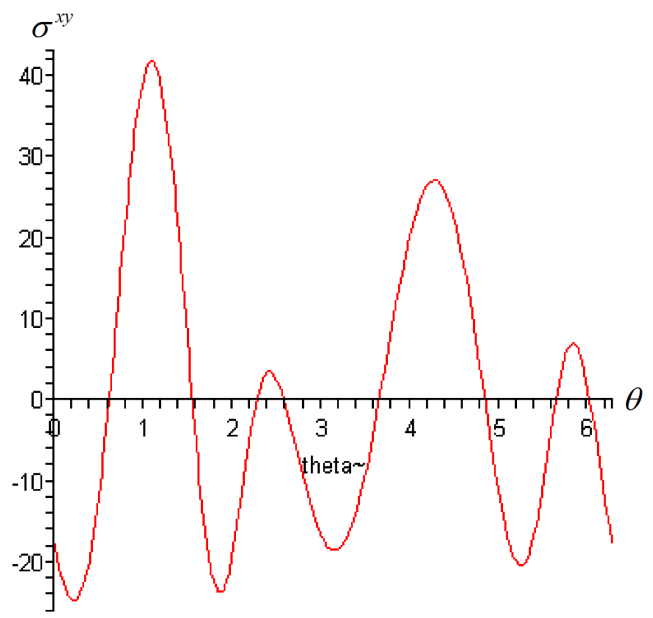

Figure 15. Maximum value of $\sigma_{x y}$ is [27.14849, [ $\left.\left.\theta=4.28087\right]\right]$, Minimum value of $\sigma_{x y}$ is [ $\left.-18.54277,[\theta=3.15247]\right]$.

738 
The complex constant $b$ has been determined by Equation (18) and its value was calculated by using Maple 12. For $n=0.1+0.1 i, m=0.2-0.2 i, d=1+i, P=1 / 4$ and $\chi=2$. The relation $\theta$ between the stress components $\sigma_{x x}, \sigma_{y y}, \sigma_{x y}$ and the angle $\theta$ are considered in Figures 16-18.

Case (2): When the curvilinear centre not allowed to rotate, i.e. when $\ell=1$ and $\varepsilon=0$. This means that the rigid curvilinear kernel in restrained in its original position by a couple which is not sufficient to rotate. The Goursat functions, in this case, become

$$
\begin{gathered}
\phi(\zeta)=\frac{1}{\chi}\left[-\frac{\bar{d} P \mathrm{e}^{2 \mathrm{i} \vartheta}}{2 \zeta}+\frac{h}{\zeta-n}\left(b+N\left(n^{\nu-1}\right)\right)\right], \\
\psi(\zeta)=\frac{\chi \bar{d} P}{4 \zeta}-\frac{\omega\left(\zeta^{-1}\right)}{\omega^{\prime}(\zeta)} \phi_{*}(\zeta)+\frac{\bar{h} \zeta}{1-\bar{n} \zeta} \phi_{*}\left(n^{-1}\right) .
\end{gathered}
$$

For $\ell=1, n=0.1+0.1 \mathrm{i}, m=0.2-0.2 \mathrm{i}, d=1+\mathrm{i}, \quad \Gamma=\frac{P}{4}, \quad \Gamma^{*}=-\frac{1}{2} P \mathrm{e}^{-2 \mathrm{i} \vartheta}, P=1 / 4$ and $\vartheta=\pi / 4, \pi \approx 3.14159$ the relation between the stress components $\sigma_{x x}, \sigma_{y y}, \sigma_{x y}$ and the angle $\theta$, using Maple 12 are considered in Figures 19-21.

4) When the force acts on the centre of the curvilinear kernel and the stresses vanish at infinity. In this case the kernel can not be rotate and it remains in its original position. Hence, we get

$$
\begin{aligned}
& \phi(\zeta)=\frac{1}{\chi} \frac{h}{(\zeta-n)}\left[b-\frac{X-\mathrm{i} Y}{2 \pi(1+\chi)} n\right], \\
& \psi(\zeta)=\frac{\bar{h} \zeta}{1-\bar{n} \zeta} \phi_{*}\left(n^{-1}\right)-\frac{\omega\left(\zeta^{-1}\right)}{\omega^{\prime}(\zeta)} \phi_{*}(\zeta),
\end{aligned}
$$

where

$$
\phi_{*}(\zeta)=\phi(\zeta)-\frac{X+\mathrm{i} Y}{2 \pi(1+\chi) \zeta} .
$$

Therefore, we have the solution of the second fundamental problem in the case when (X, Y) acts on the centre of curvilinear hole.

For $\ell=1, n=0.1+0.1 i, m=0.2-0.2 i, d=1+i, \quad \Gamma=\Gamma^{*}=0, k=\chi$ and $X=Y=10$ the relation between the stress components $\sigma_{x x}, \sigma_{y y}, \sigma_{x y}$ and the angle $\theta$, using Maple 9.5 are considered in Figures 22-24.

\section{Conclusion and Discussion}

From the previous work the following discussion and results can be concluded

1) In the theory of two-dimensional linear elasticity one of the most useful techniques for the solution of the boundary value problem for a region weakened by a curvilinear hole is to transform the region into a simpler shape to get the solution directly without difficulties.

2) The transformation mapping $z=c \omega(\zeta), \zeta=\rho \mathrm{e}^{\mathrm{i} \theta}, c$ is a complex or real constant, transforms the domain of the infinite plate with a curvilinear hole onto the domain outside (when $|\zeta|>1$ ) or into the domain inside $(|\zeta|<1)$ a unit circle.

3) The physical interest of the using mapping transform comes from its different shapes of holes it treats and different directions it takes. This mapping function deals with famous shapes of tunnels, thereon it is useful in studying the stresses around tunnels. In underground engineering the tunnel is assumed to be driven in a homogeneous, isotropic, linear elastic and pre-stressed geometrical situation. Also, the tunnel is considered to be deep enough such that the stress distribution before excavation is homogeneous. Excavating underground openings in soils and rocks is done for several purposes and in multi-sizes. At least, excavation of the opening will cause the soil or rock to deform elastically. The excavation in soil or rock is a complicated, dangerous and expensive process. The mechanics of this can be very complex. However, the use of conformal mapping that allows us to study stresses and strains around a unit circle makes it useful for engineers and easier for mathematicians. 
M. A. Abdou, A. R. Jan

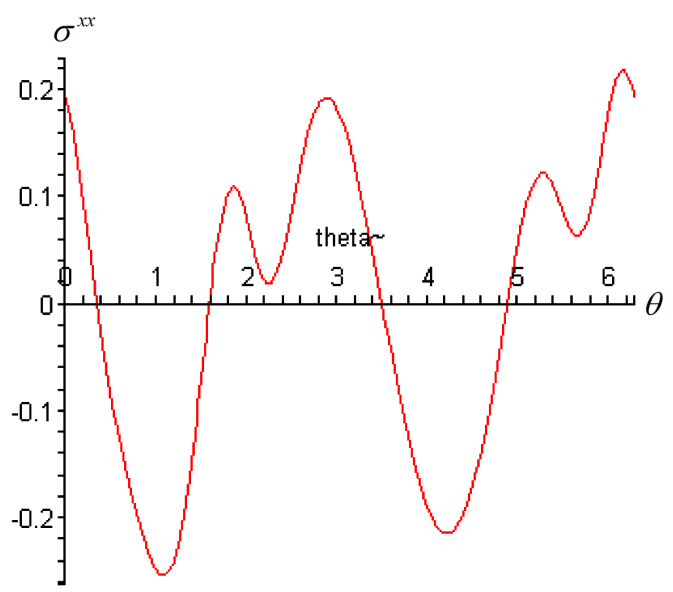

Figure 16. Max. and Min. values of $\sigma_{x x}$ are [0.19217, [ $\left.\theta=2.88015\right]$, $[-0.21424,[\theta=4.21641]]$.

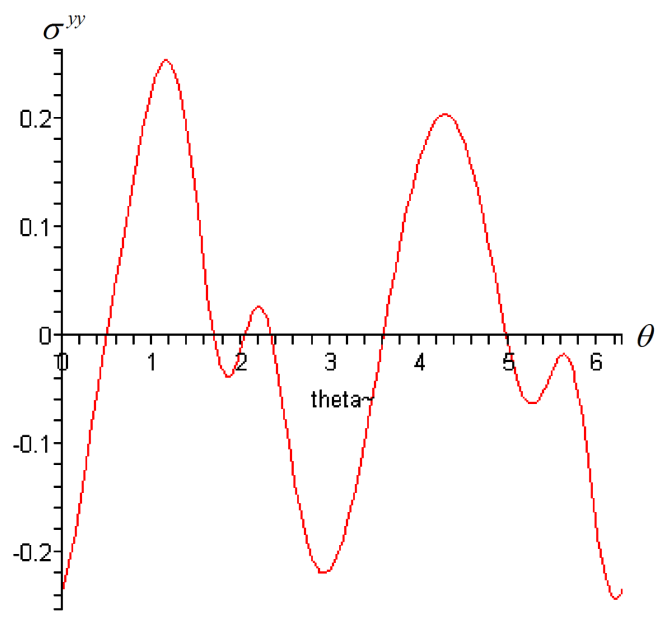

Figure 17. Max. and Min. values of $\sigma_{y y}$ are [0.20395, [ $\left.\theta=4.29250\right]$, $[-0.21998,[\theta=2.93249]]$.

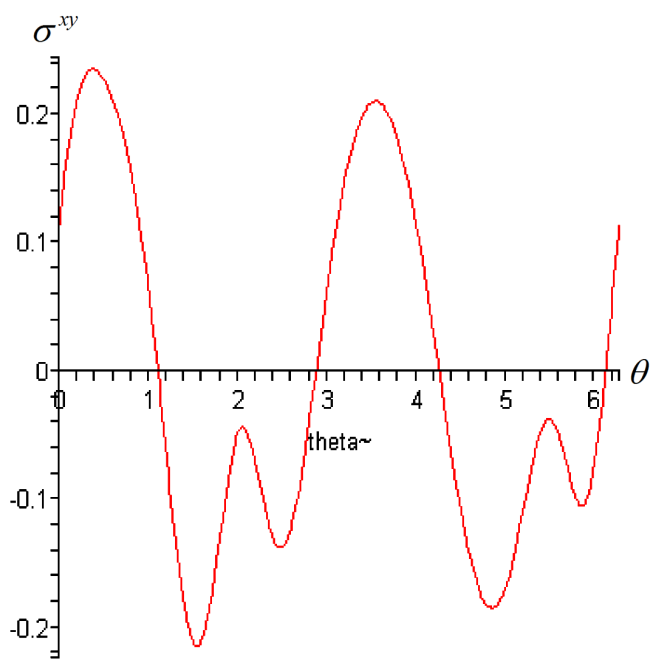

Figure 18. Maximum value of $\sigma_{x y}$ is [0.21042, [ $\left.\left.\theta=3.54667\right]\right]$, Minimum value of $\sigma_{x y}$ is $[-0.13798,[\theta=2.47646]]$.

(740) 


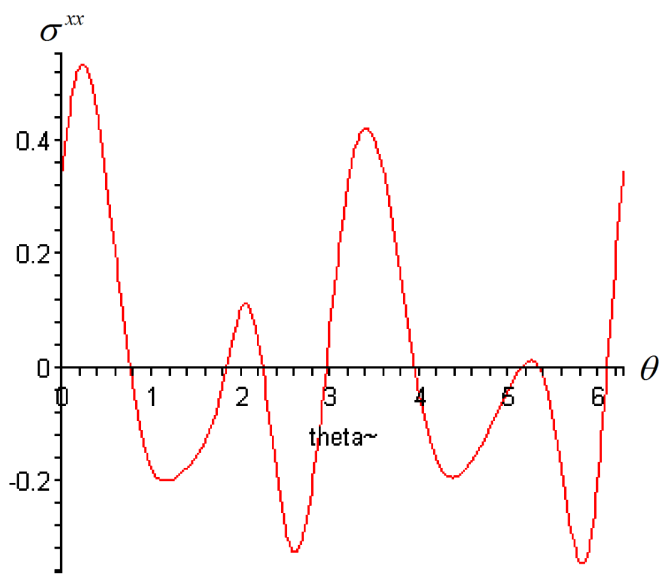

Figure 19. Max. and Min. values of $\sigma_{x x}$ are [0.42251, [ $\left.\left.\theta=3.40048\right]\right]$, $[-0.32725,[\theta=2.60422]]$.

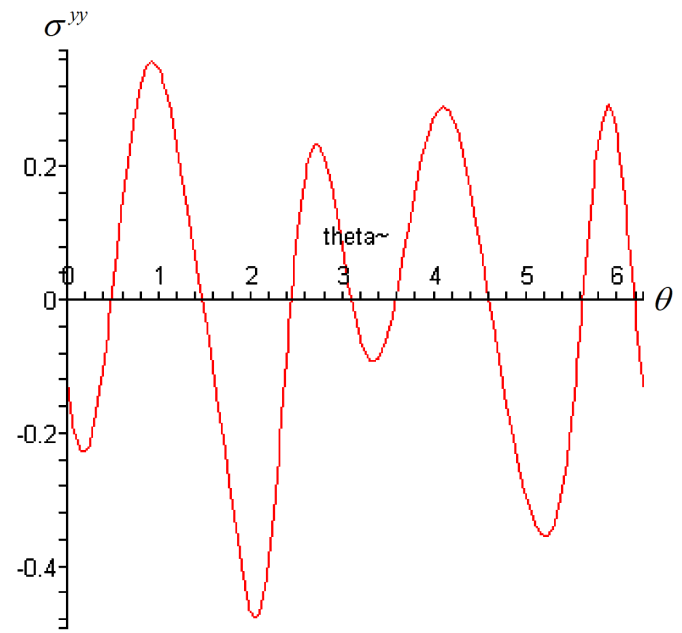

Figure 20. Max. and Min. values of $\sigma_{v v}$ are [0.29017, [ $\left.\left.\theta=4.09478\right]\right]$, $[-0.47644,[\theta=2.04499]]$.

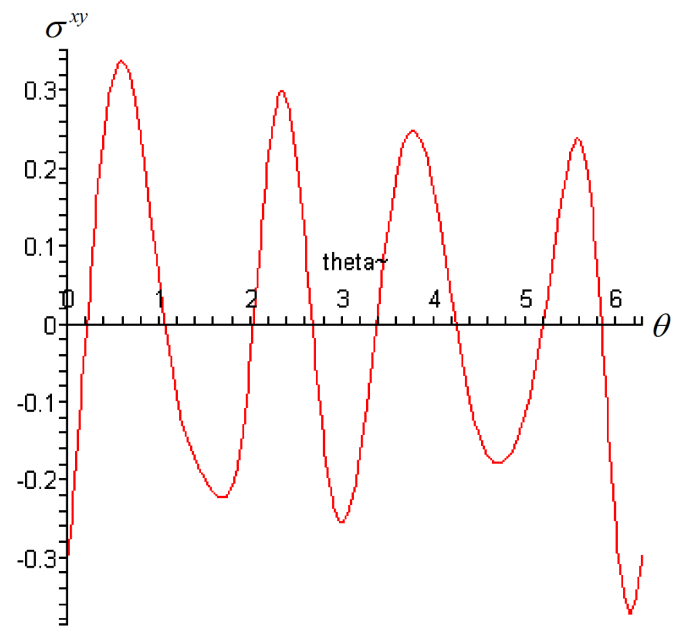

Figure 21. Maximum value of $\sigma_{x y}$ is [0.24900, [ $\left.\left.\theta=3.77559\right]\right]$, Minimum value of $\sigma_{x y}$ is $[-0.22325,[\theta=1.68785]]$. 
M. A. Abdou, A. R. Jan

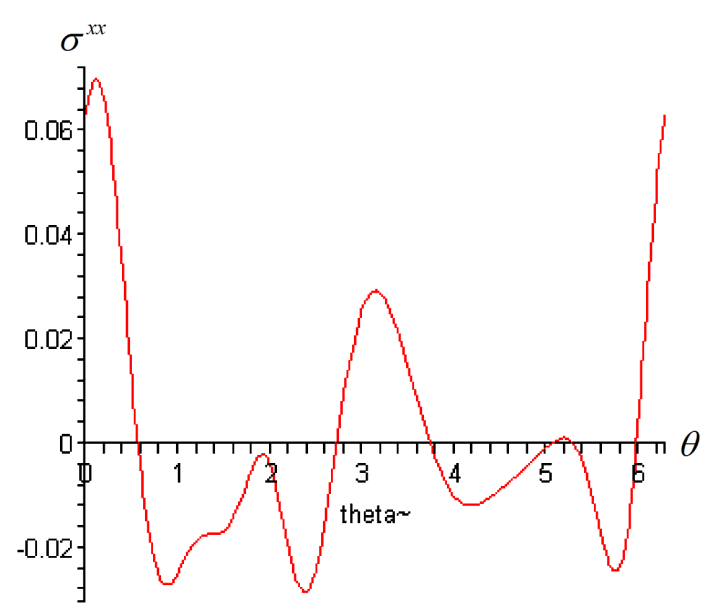

Figure 22. Max. and Min. values of $\sigma_{x x}$ are [0.06313, [ $\left.\theta=6.28319\right]$, $[-0.02863,[\theta=2.37434]]$.

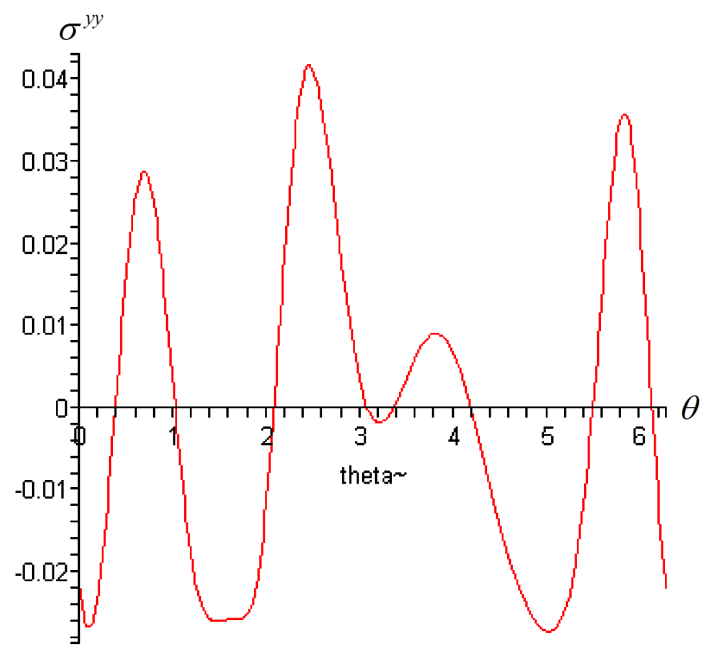

Figure 23. Max. and Min. values of $\sigma_{y y}$ are [0.04161, [ $\left.\theta=2.45943\right]$, $[-0.02730,[\theta=5.01912]]$.

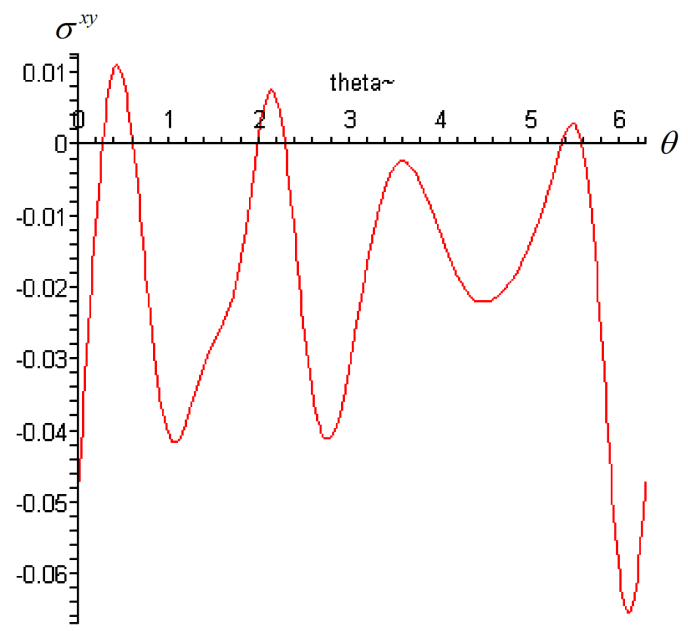

Figure 24. Maximum value of $\sigma_{x v}$ is $[-0.00237,[\theta=3.58676]]$, Minimum value of $\sigma_{x y}$ is $[-0.04160,[\theta=1.06868]]$.

(742) 
4) The complex variables method (Cauchy method) is considered one of the best methods for solving the integro differential equation, boundary value problem, of Equation (1) and obtaining the two complex potential functions, Goursat functions, $\phi(z)$ and $\psi(z)$ directly.

5) The stress is an internal force whereas positive values of it mean that stress is in the positive direction, i.e. stress acts as a tension force. On the other side, negative values of stress mean that the stress is in the negative direction, i.e. stress acts as a press force.

6) The most important issue deduced from mapping the stress components is that $\max \sigma_{x x}=-\min \sigma_{y y}$ and vice verse $\left(\min \sigma_{x x}=-\max \sigma_{y y}\right)$.

7) When $\frac{\sigma_{x x}}{\sigma_{y y}} \rightarrow 0$ the perpendicular stress on $y$-axis is the maximum value and presents the body interior resistance of treatments (like rocks for example), whereas the perpendicular stress on $x$-axis is small according to $y$-axis. Thereon, it is better to treat the problem at points determined by angles that give minimum values of $\frac{\sigma_{x x}}{\sigma_{y y}}$.

\section{References}

[1] Colton, D. and Kress, R. (1983) Integral Equation Methods in Scattering Theory. John Wiley, New York.

[2] Ya. Popov, G. (1982) Contact Problems for a Linearly Deformable Functions. Kiev, Odessa.

[3] Noda, N., Hentarski, R.B. and Tanigowa, Y. (2003) Thermal Stresses. Taylor and Francis, UK.

[4] Schinzinger, R. and Laura, P.A. (2003) Conformal Mapping Methods and Applications. Dover Publications, New York.

[5] England, A.H. (1971) Complex Variable Method in Elasticity. McGraw, London, New York.

[6] Parkus, H. (1976) Thermo Elasticity. Spring-Verlag, Berlin,

[7] Kalandiya, A.I. (1975) Mathematical Method of Two-Dimensional Elasticity. Mir Publishers, Moscow.

[8] Muskhelishvili, N.I. (1953) Some Basic Problems of Mathematical Theory of Elasticity. Noordroof, Holland.

[9] El-Sirafy, I.H. and Abdou, M.A. (1984) First and Second Fundamental Problems of Infinite Plate with a Curvilinear Hole. Journal of Mathematical and Physical Sciences, 18, 1-12.

[10] Abdou, M.A. and Khar-Eldin, E.A. (1994) An Infinite Plate Weakened by a Hole Having Arbitrary Shape. Journal of Computational and Applied Mathematics, 56, 341-361. http://dx.doi.org/10.1016/0377-0427(94)90088-4

[11] Abdou, M.A. and Khamis, A.K. (2000) On a Problem of an Infinite Plate with a Curvilinear Hole Having Three Poles and Arbitrary Shape. Bulletin of Calcutta Mathematical Society, 92, 309-322.

[12] Abdou, M.A. (2002) Fundamental Problems for Infinite Plate with a Curvilinear Hole Having Finite Poles. Applied Mathematics and Computation, 125, 177-193. http://dx.doi.org/10.1016/S0096-3003(00)00118-1

[13] Abdou, M.A., Sabbah, A.S. and Ismail, A.S. (2002) An Infinite Plate with a Curvilinear Hole and Flowing Heat. Proceeding Mathematical Physics Society Egypt, 34, 15-27.

[14] Exadaktylos, G.E. and Stavropoulou, M.C. (2002) A Closed form Elastic Solution for Stress and Displacement around Tunnels. International Journal of Rock Mechanics and Mining Sciences, 39, 905-916.

[15] Exadaktylos, G.E., Liolios, P.A. and Stavropoulou, M.C. (2003) A Semi-Analytical Elastic Stress-Displacement Solution for Notched Circular Openings in Rocks. International Journal of Solids and Structures, 40, 1165-1187.

[16] Abdou, M.A. and Asseri, S.A. (2009) Closed Forms of Gaursat Functions in Presence of Heat for Curvilinear Holes. JJournal of Thermal Stresses, 32, 1126-1148.

[17] Abdou, M.A. and Asseri, S.A. (2009) Gaursat Functions for an Infinite Plate with a Generalized Curvilinear Hole in Zeta Plane. Applied Mathematics and Computation, 212, 23-36. http://dx.doi.org/10.1016/j.amc.2009.01.079 